\title{
Crossed-ratchet effects and domain wall geometrical pinning
}

\author{
V. I. Marconi \\ Facultad de Matemática, Astronomía y Física, Universidad Nacional de Córdoba and IFEG-CONICET, X5000HUA Córdoba, Argentina
}

A. B. Kolton

CONICET, Centro Atómico Bariloche, 8400 S.C. de Bariloche, Argentina

J. A. Capitán and J. A. Cuesta

Departamento de Matemáticas and GISC, Universidad Carlos III de Madrid, ES-28911 Leganés, Spain

A. Pérez-Junquera, M. Vélez, and J. I. Martín

Departamento de Física, Universidad de Oviedo CINN, ES-33007 Oviedo, Spain

J. M. R. Parrondo

Departamento de Física Atómica, Molecular y Nuclear and GISC, Universidad Complutense de Madrid, ES-28040 Madrid, Spain

(Received 21 December 2010; revised manuscript received 19 April 2011; published 3 June 2011)

\begin{abstract}
The motion of a domain wall in a two-dimensional medium is studied by taking into account the internal elastic degrees of freedom of the wall and geometrical pinning produced by both holes and sample boundaries. This study is used to analyze the geometrical conditions needed for optimizing crossed-ratchet effects in periodic rectangular arrays of asymmetric holes, recently observed experimentally in patterned ferromagnetic films. Exact calculation as a function of the geometry of the sample and numerical simulations have been used to obtain the anisotropic critical fields for depinning flat and kinked walls in rectangular arrays of triangles. The aim is to show with a generic elastic model for interfaces how to build a rectifier able to display crossed-ratchet effects or effective potential landscapes for controlling the motion of interfaces or invasion fronts.
\end{abstract}

DOI: 10.1103/PhysRevB.83.214403

PACS number(s): 64.60.Ht, 87.85.Qr, 75.60.Ch, 47.61.-k

\section{INTRODUCTION}

The dynamics of elastic interfaces is responsible for a wide variety of physical phenomena in very different experimental systems. Prominent examples are the propagation of reaction fronts or surface growth in material science, ${ }^{1}$ cell motility and membrane dynamics in biology, ${ }^{2}$ domain walls (DW) in ferromagnetic ${ }^{3-8}$ or ferroelectric films, ${ }^{9-11}$ fluid invasion in porous media, ${ }^{12}$ contact lines of liquids menisci, ${ }^{13}$ and crack propagation. ${ }^{14,15}$ In all these cases, the presence of heterogeneities, which locally promote wandering, compete with the elasticity of the interface, giving rise to complex collective pinning effects. Understanding these effects is a challenging problem relevant from both basic and applied viewpoints.

A particularly interesting case of interface pinning is the "geometrical pinning" induced by the presence of artificially introduced holes or antidots, ${ }^{16-18}$ or by a spatial modulation of the sample boundary conditions in narrow samples. ${ }^{7,19-23}$ These kind of boundaries can pin the interface by locally reducing its extension, thus saving surface tension energy. For extended DW this kind of pinning has been recently realized experimentally and showed to be able to modify the magnetization dynamics ${ }^{7,16,18-20}$ and to produce, in particular, interesting ratchet transport of magnetic DW. ${ }^{3,24}$ Being mostly geometrical (i.e., determined mostly by the shape and distribution of holes or by the geometry of the boundaries and not much by the specific microscopic pinning interaction) this kind of pinning has the advantage over other artificial pinning mechanisms that it can be more easily tailored at a wide range of scales to control the wall motion in various specific ways.

We have recently analyzed, specifically, the pinning effect of asymmetric holes on the propagation of DW in magnetic films, finding that, under certain geometries and oscillating external magnetic fields, the motion of flat and kinked walls is rectified in opposite directions: ${ }^{3}$ the asymmetry between forward and backward flat wall propagation results in a direct ratchet effect whereas the asymmetry between upward and downward kink propagation along a wall induces an inverted ratchet effect. This striking sensitivity yields new strategies to control the motion of the wall. The crossed rectification reported in Ref. 3 relies on the difference between the critical fields to depin the wall in each direction, and it is also present in a generic model for elastic interfaces: the $\phi^{4}$ model. ${ }^{25}$ In this paper we calculate the depinning field of a generic $\phi^{4}$ interface in the presence of an array of triangular antidots by both geometrical considerations and numerical simulations. We use this simple model because it is the minimum model that captures the essential physics behind the crossed-ratchet effects reported in Ref. 3: the competition between pinning and driving forces on a one-dimensional (1D) elastic interface that propagates in a two-dimensional (2D) array of asymmetric pinning centers. Actually, numerical simulations using the complete micromagnetic formulation provide qualitatively the same results. ${ }^{24}$ In addition, our method can be widely used to design interface rectifiers of elastic interfaces by using holes or boundary conditions in an arbitrary geometry. 
Our starting point is the overdamped $\phi^{4}$ model in the plane, i.e., a scalar field $\phi(x, y ; t)$ obeying the following evolution equation:

$$
\eta \partial_{t} \phi=c \nabla^{2} \phi+\epsilon_{0}\left(\phi-\phi^{3}\right)+H
$$

where $c$ is the elastic stiffness of the order parameter, $\epsilon_{0}$ is proportional to the local barrier separating two minima of the local free energy, $H$ is an external field biasing one of the two minima, and the friction coefficient $\eta$ sets the microscopic time scale. The evolution equation (1) derives from the energy functional

$$
E=\int d x d y\left[U(\phi(x, y))-H \phi(x, y)+\frac{c}{2}|\nabla \phi(x, y)|^{2}\right],
$$

with $U(\phi)=\epsilon_{0}\left(\phi^{2}-1\right)^{2} / 4$. In a ferromagnetic material, the first term $U(\phi)$ would correspond to an uniaxial anisotropy energy, the second term would be the Zeeman energy, and the last one would represent the exchange energy. Thus, $H$ is the magnetic field applied along the easy anisotropy axis and $\phi(x, y ; t)$ corresponds to $M / M_{S}$, the magnetization component parallel to the applied magnetic field normalized by the saturation magnetization. For $H=0$, and with the appropriate boundary condition, say, $\phi(-L, y ; t)=1$ and $\phi(L, y ; t)=-1$, the stationary solution of Eq. (1) is given by a domain on the left side of the plane with a positive and approximately homogenous field and a domain on the right side with a negative field, both separated by an interface of width proportional to $\sqrt{c / \epsilon_{0}}$. When the field is switched on to a positive (negative) value, the interface is pushed to the right (left) to minimize the total energy. However, the interface also has an elastic energy proportional to its length. Therefore, if the geometry where the field is defined is such that the length of the interface increases when moving to the left or right, then the interface will be pinned until the field reaches a critical value. Our goal is to provide an estimation of such a depinning field in a general geometry and to analyze the geometrical conditions in which rectification effects appear in the elastic interface propagation.

The organization of the paper is as follows. In Sec. II, we develop a general theoretical framework to address the problem of rectification of DW. We reduce the field $\phi$ to an elastic wall and derive an analytical expression for the local depinning field in two-dimensional stripes with arbitrarily shaped borders. In Sec. III we apply the previous results to build a 2D array of triangular holes that displays ratchet effects and give specific predictions for the appearance of normal and crossed-ratchet effects. In Sec. IV we summarize our results.

\section{DEPINNING FIELDS IN ARBITRARY GEOMETRY: GENERAL THEORY}

\section{A. From field equations to elastic walls}

Our aim is to calculate the depinning field of certain interfaces in an arbitrary geometry. To simplify the task, we first need to reduce the whole field equation (1) to a parametric description of the interface, in the spirit of the collective coordinate approach widely used in one-dimensional models. ${ }^{26}$
In Appendix A we construct a solution of Eq. (1) where two domains of positive and negative magnetization are separated by a wall defined by the line $[x(s), y(s)]$. The solution reads

$$
\phi(x, y)=\tanh \left[\frac{g(x, y)}{w}\right],
$$

where $g(x, y)$ is the distance of point $(x, y)$ to the backbone of the wall $[x(s), y(s)]$ and

$$
w=\sqrt{\frac{2 c}{\epsilon_{0}}}
$$

can be considered as its width. Equation (3) is well known as the field corresponding to a single wall in the one-dimensional $\phi^{4}$ model, and, as we show in Appendix A, can be extended to two dimensions if the curvature radius of the wall $[x(s), y(s)]$ is much larger than its width $w$.

The energy of this solution, for small width $w$, can be approximated by (see Appendix A)

$$
E=\sigma d-2 H A,
$$

where $d$ is the length of the wall $[x(s), y(s)], A$ is the area of the positive domain (at one side of the wall), and

$$
\sigma=\frac{\sqrt{8 \epsilon_{0} c}}{3}
$$

is the energy of the wall per unit of length. Consequently, the wall behaves as an elastic line with a linear tension $\sigma$ and pushed by a field $H$.

Our approximations are exact for infinitely narrow interfaces, $w \rightarrow 0$, since we are reducing the field in the whole plane to a single curve defining the center of the interface. For thin interfaces the approximation is good enough, provided the width of the wall remains approximately constant all along the curve and that the local curvature radius of the line is smaller than the domain wall width $w$. In brief, these conditions ensure that the state of $\phi$ with a domain wall can be well described exclusively by the transverse degrees of freedom of an elastic interface.

\section{B. Interface pinning in a holed medium}

We will now consider a domain wall in a two-dimensional medium with holes or multiply connected space. For the scalar field $\phi$ this amounts to solving Eq. (1) in a domain $\Omega-\Delta$, which includes all the two-dimensional space $\Omega$, except the possibly noncompact region $\Delta$ occupied by the holes and outer space. The boundary conditions at the border of the holes, $\delta \Delta$, depend on the specific physical system modeled by Eq. (1). Throughout this paper we set free (Neumann) boundary conditions for the order parameter, i.e., $\left.\partial_{\mathbf{n}} \phi\right|_{\delta \Delta}=0$. This is an appropriate choice if $\phi$ models the magnetization of a material along a given direction and the holes $\Delta$ are simply defined as the absence of magnetic material. The simplest picture of this situation is a set of discrete spins with no interaction with the hole: the magnetization $\phi$ can take any value at the border $\delta \Delta$, but the absence of an interaction term with the hole implies the Neumann condition $\left.\partial_{\mathbf{n}} \phi\right|_{\delta \Delta}=0$. A consequence of this type of boundary condition is that the DW are orthogonal to the boundaries [see below Eq. (8)], 


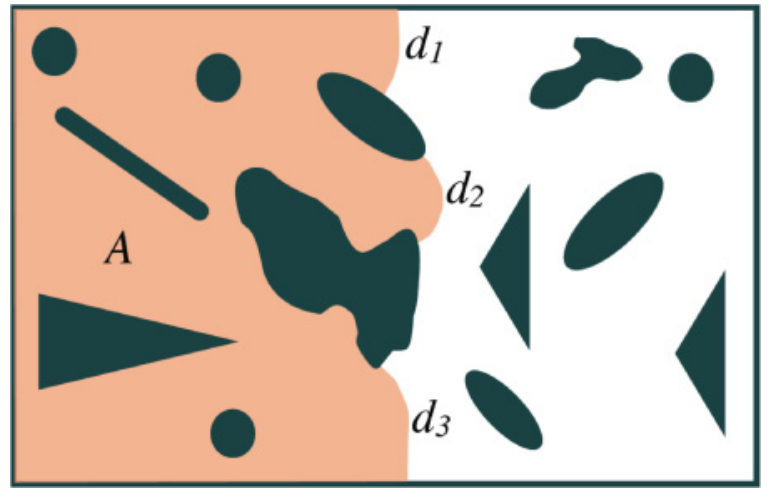

FIG. 1. (Color online) Interface in a two-dimensional medium with free holes (shapes in black/dark green) and sample boundaries (black/dark green rectangle). The gain of interface energy by optimally intersecting the holes and sample boundaries produces domain wall pinning.

in agreement with the walls observed by full micromagnetic calculations. ${ }^{24}$ Neumann boundary conditions are suitable for other applications of the $\phi^{4}$ Eq. (1), such as reaction diffusion equations where $\phi$ represents the concentration of a reactant confined in $\Omega-\Delta$.

Within the interface approximation described in Sec. II A, interface pinning arises from the gain of line energy (reduction of the total length of the interface) that is possible by optimally intersecting the holes and sample boundaries (see Fig. 1). From Eq. (5) the energy of the pinned domain wall then reads

$$
E=\sigma \sum_{i=0}^{N} d_{i}-2 H A,
$$

where $d_{i}=\int_{s_{i}}^{s_{i+1}} d s \sqrt{\dot{x}^{2}+\dot{y}^{2}}$ is the length of the interface segment connecting the holes $i$ and $i+1$ (with $i=0$ and $i=N$ designating the sample boundaries) and the area $A$ now excludes regions belonging to $\Delta$.

The free (Neumann) boundary conditions for the order parameter at the hole and sample boundaries translate in the interface description in the orthogonality condition

$$
\mathbf{v}_{i} \cdot \mathbf{t}_{i}=0 \quad \forall i,
$$

where $\mathbf{t}_{i}$ is the tangent vector of the boundary and $\mathbf{v}_{i} \equiv$ $\left[\dot{x}\left(s_{i}\right), \dot{y}\left(s_{i}\right)\right]$ is the tangent vector of the interface, both at the intersection point $\left[x\left(s_{i}\right), y\left(s_{i}\right)\right]$.

Metastable states of the interface are therefore local minima of the energy (7) with segments satisfying the orthogonality constraint (8) at its ends. In the following we discuss the geometry of these optimal segments, which are the building blocks of our method.

\section{Equilibrium state of a wall}

Our next step is to calculate the equilibrium profile of an interface segment and its stability. The energy of an interface segment is a function of both its shape and the location of its ends or contact points. In order to find the possible metastable states of the segment we need to minimize the energy given by Eq. (5) with the constraint (8).

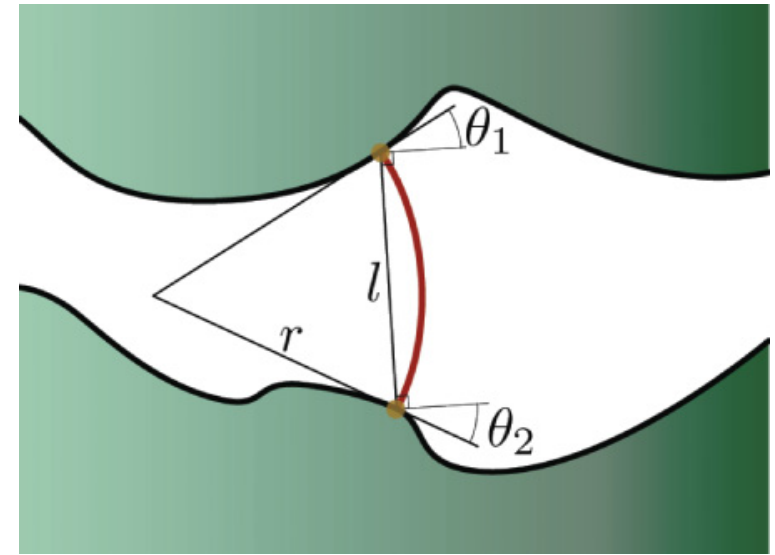

FIG. 2. (Color online) The energy of an elastic wall (dark grey arc or red arc online) is minimized by an arc of radius $r=\sigma /(2 H)$. An equilibrium state is reached when the arc intersects the boundaries orthogonally.

As shown in Appendix B, the solution of the corresponding Euler-Lagrange equation, regardless of any boundary condition, is a circular arc of radius

$$
r \equiv \frac{\sigma}{2 H}
$$

Consider now a wall confined between two irregular boundaries, as plotted in Fig. 2. Let $l$ be the distance between the contact points, and let $\theta_{1}+90^{\circ}\left(\theta_{2}+90^{\circ}\right)$ be the angle formed by the upper (lower) boundary and the line connecting the two contact points. The elastic wall minimizes its energy by adopting the shape of an arc of radius $r$ and it must be orthogonal to the boundaries at the contact points. As illustrated in Fig. 2, this implies that $\theta_{1}=\theta_{2}=\theta$ and

$$
\sin \theta=\frac{l / 2}{r}=\frac{H l}{\sigma}
$$

where we have used the expression for the radius of the wall, Eq. (9).

\section{Local depinning fields for an anchored wall}

We can now proceed to calculate local depinning fields for narrow DW bounded between two borders, which are central for studying the ratchet effect. Given a metastable state of the anchored domain wall the local depinning field is defined as the maximum field it can support by deforming continuously as we increase the field. Above this local depinning field the domain wall escapes the local environment and slides until it is trapped again in a new metastable state with a larger depinning field, if it exists. ${ }^{27}$ Otherwise it continues sliding.

As an illustration, consider the particular case where the bottom border is the $x$ axis and the top border is given by and arbitrary smooth function $f(x)>0$. The wall, as we have seen above, is an arc of radius $r=\sigma /(2 H)$. Its center must lie on the $x$ axis, say at $x_{0}$, since the wall is perpendicular to the $x$ axis at the lower contact point. The upper contact point, $\left[x_{1}, f\left(x_{1}\right)\right]$, belongs to the arc, and hence $\left(x_{1}-x_{0}\right)^{2}+f\left(x_{1}\right)^{2}=r^{2}$, and the orthogonality condition implies

$$
\frac{f\left(x_{1}\right)}{x_{1}-x_{0}}=f^{\prime}\left(x_{1}\right)
$$


The upper contact point is then given by the condition

$$
r=\frac{f\left(x_{1}\right)}{f^{\prime}\left(x_{1}\right)} \sqrt{1+f^{\prime}\left(x_{1}\right)^{2}} .
$$

Note that only the solutions with $f^{\prime}\left(x_{1}\right) \geqslant 0$ must be taken if $H \geqslant 0$. If such a solution $x_{1}$ exists for a given $r$ (i.e., for a given field $H$ ), the lower contact point is given by $x_{2}=x_{1}$ if $f^{\prime}\left(x_{1}\right)=0$ and $x_{2}=r+x_{1}-f\left(x_{1}\right) / f^{\prime}\left(x_{1}\right)$ otherwise.

For $H=0(r \rightarrow \infty)$ the only possible solutions are points $x_{1}$ such that $f^{\prime}\left(x_{1}\right)=0$. These solutions are straight vertical segments joining the two borders at $x_{1}$. For concreteness let us assume that $x_{1}=0$ for $H=0$ and that $f^{\prime \prime}(0)>0$, so the initial state is metastable. In such a case if we quasistatically increase $H$ (decrease $r$ ) from $x_{1}=0$ we can generate a continuum set of solutions $x_{1}(r)$ parametrized by the field. At some field $H_{c}=\sigma / 2 r_{c}$ it is possible however to have a discontinuity in $x_{1}(r)$ due to the absence of solutions beyond $H_{c}$. We can then define the critical radius of the initial metastable state as

$$
r_{c}=\min _{x_{1}}\left\{\frac{f\left(x_{1}\right)}{f^{\prime}\left(x_{1}\right)} \sqrt{1+f^{\prime}\left(x_{1}\right)^{2}}\right\}_{f^{\prime}\left(x_{1}\right)>0},
$$

where the condition $f^{\prime}\left(x_{1}\right)>0$ ensures that $r_{c}$ is positive, so we obtain the forward depinning field. The depinning field is therefore $H_{c}=\sigma / 2 r_{c}$, the upper contact point of the critical arc is $x_{c}^{\text {up }} \equiv x_{1}\left(r_{c}\right)$, and the lower contact is $x_{c}^{\text {low }}=r+x_{c}-$ $f\left(x_{c}\right) / f^{\prime}\left(x_{c}\right)$.

As an illustration consider the geometry displayed in Fig. 3, $f(x)=2-\cos (x)$. As a function of the field $H$ stable arcs have a radius $r=\sigma / 2 H$ and the upper contact point must satisfy

$$
r=\frac{2-\cos (x)}{\sin (x)} \sqrt{1+\sin (x)^{2}} .
$$

The solutions of this equation for different fields are shown graphically in Fig. 3 (upper plot), and the corresponding arcs are shown in Fig. 3 (lower plot). The critical state [also shown in Fig. 3 (lower plot)] has $x_{c}^{\text {up }} \approx 0.85$ and corresponds to $r_{c} \approx$ 2.23 and $x_{2}^{\text {low }} \approx 1.30$. The initially flat interface for $H=0$ will shift forward quasistatically upon increasing the field, following the $x_{1}(r)$ curve. Above $H_{c}=\sigma /\left(2 r_{c}\right)$ the interface will move at a finite speed.

Let us now analyze the depinning from a rounded tip of curvature radius $W$, as shown in Fig. 4. We assume, for concreteness, the form

$$
\begin{gathered}
f(x)=\left(l_{0} / 2+W\right)-\sqrt{W^{2}-x^{2}}, \quad|x|<x_{0}, \\
f(x)=f\left(x_{0}\right)+\tan \alpha\left|x-x_{0}\right|, \quad|x|>x_{0},
\end{gathered}
$$

where $x_{0}=W \sin (\alpha)$ and $f\left(x_{0}\right)=\left(l_{0} / 2+W\right)-W \cos \alpha$. The first equation describes a rounded circular point, and the second a line with the asymptotic slope angle $\alpha$. In this case $x_{1}$ increases monotonically from zero and no more solutions of Eq. (11) exist for $x>x_{0}$. We thus have $x_{c}=W \sin \alpha$ and $r_{c}=f\left(x_{0}\right) / \sin \alpha$. The critical field is therefore

$$
H_{c}=\frac{\sigma \sin \alpha}{l_{0}+2 W(1-\cos \alpha)} .
$$

For a sharp $W \rightarrow 0$ tip we have

$$
H_{c}=\frac{\sigma \sin \alpha}{l_{0}},
$$

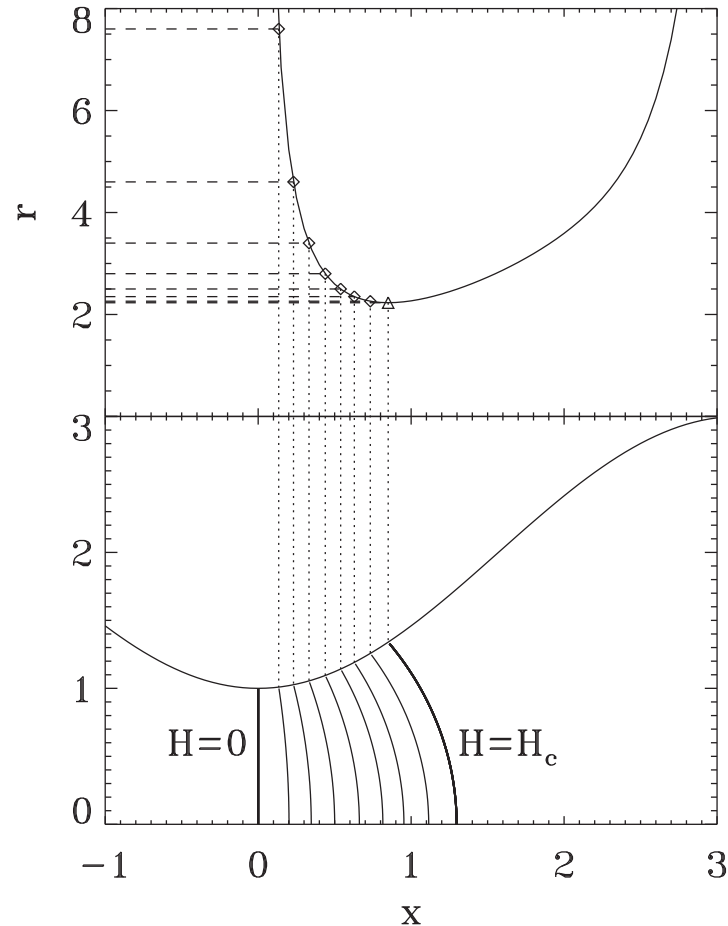

FIG. 3. Construction of the metastable elastic lines bounded between 0 and $f(x)=2-\cos (x)$, as a function of the external field or arc radius. We depict the boundaries and the metastable walls for different fields $H$ in the lower plot. In the upper plot we represent the function $\left[f(x) / f^{\prime}(x)\right] \sqrt{1+f^{\prime}(x)^{2}}$. The intersection of the arc radii $r=\sigma /(2 H)$ with this function gives the upper contact point $\left[x_{1}, f\left(x_{1}\right)\right]$ of the metastable wall with the top border $f(x)$. The depinning field is $H_{c}$, above which there are no metastable states.

implying a very strong pinning in the limit of strong constriction $l_{0} \rightarrow 0$. Interestingly, in this limit the depinning field would be ultimately controlled by the rounding $W$ in more realistic rounded tips.

\section{BUILDING A 2D RATCHET}

From this general theory of interface pinning, we will show how to build a $2 \mathrm{D}$ ratchet for extended DW with both direct and inverted rectification effects, as a function of the

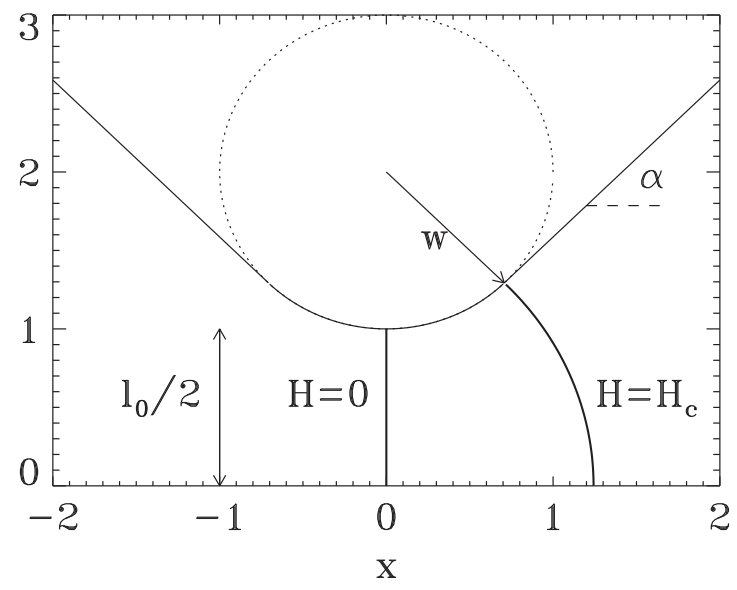

FIG. 4. A tip rounded at the scale $W$. 


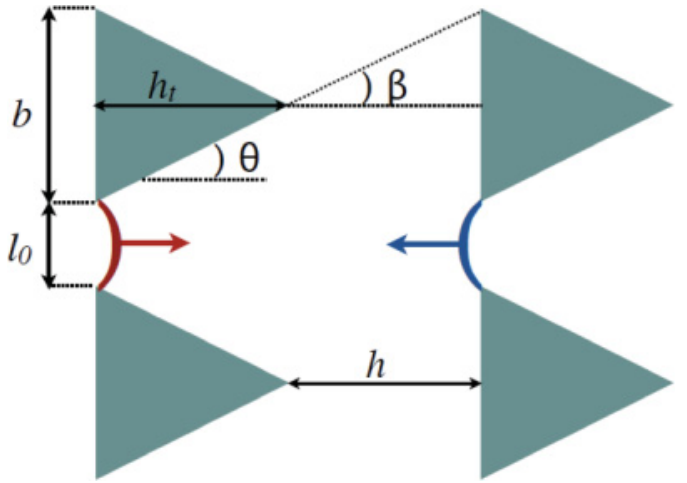

FIG. 5. (Color online) A two-dimensional ratchet geometry made by triangles. The arcs are a schematic representation of a domain wall moving either to the right or to the left. These two directions of propagation are not equivalent since the wall must be orthogonal to the boundaries at the contact points: this implies a different configuration for propagation to the left along the vertical triangle bases than for propagation to the right along the diagonal triangle sides. If the holes would be symmetric (circles or squares) both directions of motion would be the same, without obtaining a ratchet geometry.

applied field, by choosing the appropriate geometry for an array of asymmetric holes. We take as a starting point the geometry depicted in Fig. 5, where triangular defects are distributed in a rectangular array, which is similar to the hole arrangement analyzed in Ref. 3. This array of triangles presents a symmetry of reflection along the $X$ axis but broken reflection symmetry along the $Y$ axis, which is the basic condition for the observation of ratchet effects: the equivalence between forward and backward domain wall propagation is broken in the array, allowing for domain wall rectification effects.

This kind of ratchet effect has been mostly studied in 1D magnetic nanowires. ${ }^{19-22}$ However, in a 2D array such as shown in Fig. 5 the 1D character of the elastic domain walls opens the possibility of extra propagation modes. In particular, when a wall is pinned between two lines of defects, it develops kinks and antikinks, as shown in Fig. 6. Kinked walls have already been observed by magnetooptical Kerr effect microscopy and micromagnetic simulations in magnetic films with 2D arrays of asymmetric defects. ${ }^{3,24}$ In general, kinks can be nucleated as soon as there is a point in the line of defects with a different depinning field from the rest. In real magnetic films of finite area this can happen either at the film boundary (the wall segment located between the last hole in the array and the film boundary being different from any other wall segment pinned in between two triangles) or due to small inhomogeneities in the sample.

Depending on their shape and the sign of the field, kinks and antikinks can move upward or downward, turning in a net wall motion to the left or to the right. This kink motion is also asymmetric, reflecting the $Y$-axis asymmetry of the pinning potential by the array of triangles, so that it opens the possibility of a rectified motion of the kinked wall. Let us analyze in detail how the symmetry properties of the array influence kink propagation: for example, as shown in Fig. 6, a kink moving upward [Fig. 6(a)] is equivalent to an antikink moving downward [Fig. 6(b)] upon reflection along
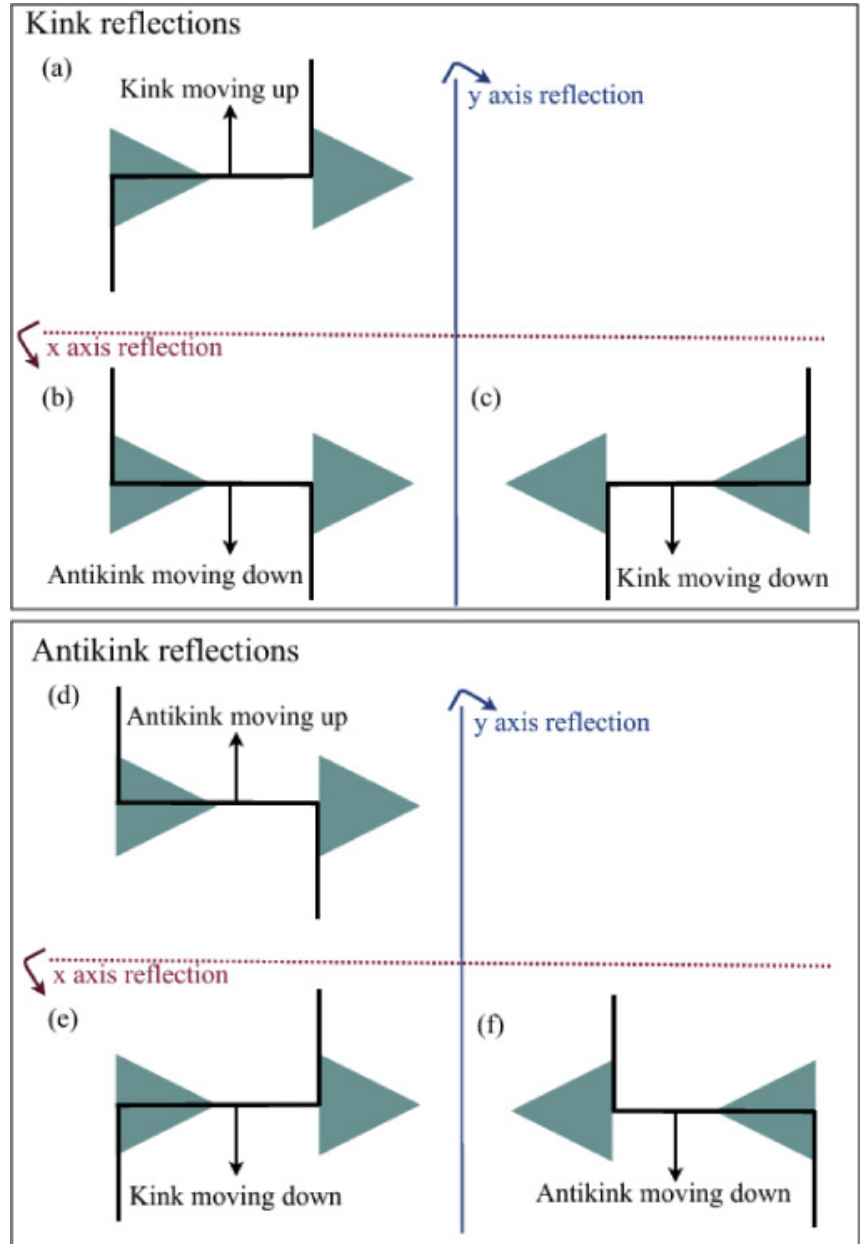

FIG. 6. (Color online) Broken symmetry in a 2D kinked wall. (a) Kink moving upward. (b) After an $x$-axis reflection, an antikink moving downward is obtained. (c) After a second $y$-axis reflection, a kink moving downward on a different array (inverted triangle array) is obtained. From (d) to (f) the same reflections are shown for an antikink.

the $X$ axis, which is an allowed symmetry operation of the array of triangles. In fact, both movements (kink upward and antikink downward) result in a net backward motion of the extended domain wall. The critical field for this propagation process will be labeled $H_{\mathrm{U}}$ from now on. However, a kink moving downward [Fig. 6(c)] is the result of a reflection upon the $Y$ axis of the antikink moving downward. This is a broken symmetry in the array (note the inverted triangles), implying that both situations are not equivalent. Actually, both the downward motion of a kink [Fig. 6(e)] and the upward motion of an antikink [Fig. 6(d)], which are equivalent upon reflection along the $X$ axis, result in a net forward motion of the extended wall. The critical field for this propagation process will be labeled $H_{\mathrm{D}}$ in the following. In short, $H_{\mathrm{U}}$ and $H_{\mathrm{D}}$ could not be the same due to the broken $Y$-axis symmetry in the array.

Thus, to understand domain wall propagation in the rectangular array of triangles two facts must be considered: first, the broken $Y$-axis symmetry breaks the equivalence between forward and backward domain wall propagation; second, the extended nature of DW in the 2D array of holes allows for 
extra propagation modes not possible in 1D geometries, such as those corresponding to nanowires: flat wall propagation and kinked wall propagation. If a wall is pushed to the right by the applied field two different propagation modes can be activated: either forward flat wall propagation (at a critical field $H_{\mathrm{F}}$ ) or kinked wall propagation (by kinks moving downward and/or antikinks moving upward at $H_{\mathrm{D}}$ ). In contrast, if a wall is pushed to the left by the applied field the possible propagation modes will be either backward flat wall propagation (at a critical field $H_{\mathrm{B}}$ ) or kinked wall propagation (by kinks moving upward and/or antikinks moving downward at $H_{\mathrm{U}}$ ). The global behavior of walls upon propagation across the array of asymmetric defects will depend on the relationships among the four relevant critical fields $H_{\mathrm{F}}, H_{\mathrm{B}}, H_{\mathrm{U}}$, and $H_{\mathrm{D}}$. Actually, for some geometries, it can happen that the rectification of kink motion is opposite to the rectification of a vertical nonkinked wall described previously. This crossed-ratchet effect offers promising technological applications since it allows a nontrivial control of the two-dimensional wall that provides, for example, a memory effect in the magnetic system: ${ }^{3}$ once a wall has propagated into the patterned film pushed in the forward direction by a field in the range $H_{\mathrm{F}}<H<H_{\mathrm{B}}$, it will move backward under the action of an ac field of amplitude $H<H_{\mathrm{U}}$. Thus the magnetization relaxes toward its last saturating value and the system remembers the sign of the last saturating magnetic field independent of the value of the remanent magnetization.

First, we will analyze the propagation of a flat wall crossing a line of triangular defects (forward-backward ratchet), which is equivalent to the propagation of DW in nanowires with asymmetric geometry. ${ }^{19-22}$ Then, we will study the upwarddownward propagation of a kink in a wall pinned in between two adjacent defect lines (upward-downward ratchet). Finally, we will discuss the geometrical parameters needed to design 2D arrays of asymmetric holes with crossed-ratchet behavior (with opposite sign for forward-backward and upwarddownward ratchets).

\section{A. Flat walls: Forward and backward propagation}

An infinitely narrow domain wall moving from the left to the right (forward) across a vertical line of triangles (see Fig. 5) will be pinned at the base of the triangles, where the distance between the ends of the wall is minimum. The depinning field can be derived from the one obtained in Sec. II D for a geometry defined tip and a flat boundary, as in Fig. 4. Now we have two symmetric tips but this situation is equivalent to the previous one if we add the mirror image of the tip, with the wall adopting the same shape as the one depicted in Fig. 4 (plus its mirror image). Therefore, the depinning field is identical to the one given by Eq. (17):

$$
H_{\mathrm{F}}=\frac{\sigma \sin \theta}{l_{0}},
$$

where $\theta$ is the angle between the sides of the triangles and the horizontal, and $l_{0}$ is the vertical distance between triangles (see Fig. 5). Notice also that the critical field (18) is, according to Eq. (10), the one for which the wall accommodates to the boundary of both triangles.
A wall moving to the left (backward), like the one depicted in Fig. 5, will also be pinned between the same vertices of the triangles, but now it has to grow along the vertical bases, i.e., the angle of the boundaries in Eq. (17) is $90^{\circ}$. Therefore,

$$
H_{\mathrm{B}}=\frac{\sigma}{l_{0}} .
$$

We find $H_{\mathrm{F}} / H_{\mathrm{B}}=\sin \theta \leqslant 1$, i.e., it is easier for the wall to move forward than backward, as expected. The triangles can therefore rectify the motion of the wall. By applying an alternating field of peak intensity $H$, with $H_{\mathrm{B}}<H<H_{\mathrm{F}}$, the wall will have a net forward motion, so that a direct ratchet effect is obtained.

Finite width effects: We next compare our simple previous geometrical estimates for $H_{\mathrm{F}}$ and $H_{\mathrm{B}}$ with simulations for a more realistic $\phi^{4}$ domain wall with a finite width, as was done in Ref. 3. We integrate the evolution equation, Eq. (1), using typical first-order integration methods. ${ }^{28}$ We use a rectangular $L_{x} \times L_{y}$ box with periodic boundary conditions in the $y$ direction and we impose the presence of an initial DW fixing the following boundary conditions in the $x$ direction: $\phi((x=$ $0), y, t)=1$ and $\phi\left(\left(x=L_{x}\right), y, t\right)=-1$. The simulations then start with a flat wall at the left border of the sample, where no holes are present. An array of asymmetric holes (triangles) in the center of the sample, with the parameters depicted in Fig. 5, is introduced in the model, imposing free boundary conditions for the scalar order parameter at the holes border, as explained in Sec. II B. Then the system response to positive and negative applied fields can be probed and the depinning magnetic fields can be obtained and fully characterized versus all geometrical parameters $\left(l_{0}, b, \theta, \beta, h\right)$ and material properties $\left(c, \epsilon_{0}, \omega, \sigma\right)$.

In the inset of Fig. 7 we plot the backward depinning field, $H_{\mathrm{B}}$, of a $\phi^{4}$ domain wall as a function of $l_{0}$ for different elastic constants $c$ and fixed $\epsilon_{0}=1$. This is equivalent to changing the domain wall width $w=\sqrt{2 c / \epsilon_{0}}$ and the domain wall

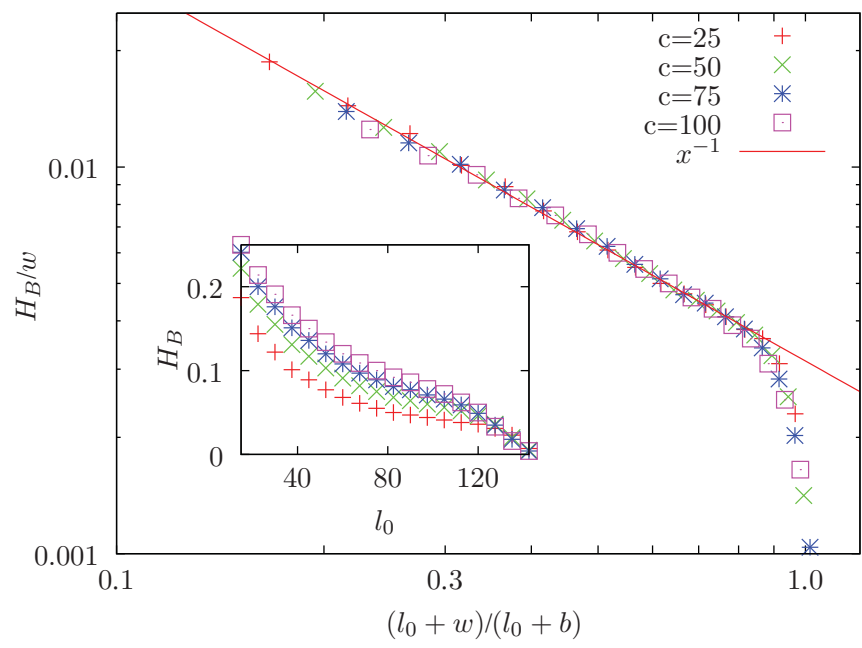

FIG. 7. (Color online) Scaled $H_{\mathrm{B}} / w$ vs $\left(l_{0}+w\right) /\left(l_{0}+b\right)$ for different values of $c, l_{0}$, and $b$. The solid line indicates the $H_{\mathrm{B}} / w \sim$ $\left(l_{0}+w\right)^{-1}$ dependence. The inset shows a backward depinning field for a $\phi^{4}$ domain wall, $H_{\mathrm{B}}$, as a function of the vertical gap between triangles, $l_{0}$, for different values of the elastic constant $c$ or domain wall width $w$. 
energy $\sigma=\sqrt{8 c \epsilon_{0}} / 3$, which scales as $\sigma \sim w . H_{\mathrm{B}}$ is found to increase as a function of $c$, mainly due to the increase in domain wall energy $\sigma$, and to decrease as a function of $l_{0}$. All the data of $H_{\mathrm{B}}$ obtained from the different simulations can be scaled to a single curve, if we plot $H_{\mathrm{B}} / w \sim H_{\mathrm{B}} / \sigma$ as a function of $\left(l_{0}+w\right) /\left(l_{0}+b\right)$ (see main panel of Fig. 7). For small values of $l_{0}, H_{\mathrm{B}} / w \sim\left(l_{0}+w\right)^{-1}$ as predicted by Eq. (19), except for a correction to $l_{0}$ which is of the order of the domain wall width $w$. This correction can be qualitatively understood by noting that the $\phi^{4}$ wall just below $H_{\mathrm{B}}$ extends from the tip of the triangles up to a distance of order $w$ into the base of each of them, so that the center of the wall describes an arc covering a vertical distance $l_{0}+w$. It is interesting to mention that this correction is the same as predicted by Eq. (16) for a rounded tip of curvature radius equal to domain wall width ( $W=w$ ) and $\alpha=90^{\circ}$ (corresponding to backward depinning). That is, finite domain wall width and tip rounding of defect geometry have equivalent effects on depinning fields, softening the magnetic behavior in comparison to an analytical calculation for sharp tips and narrow DW. At large values of $l_{0}, H_{\mathrm{B}} / w$ deviates from the behavior $H_{\mathrm{B}} / w \sim\left(l_{0}+w\right)^{-1}$, decreasing steeply precisely when $\left(l_{0}+w\right) /\left(l_{0}+b\right) \sim 1$. The reason is that for the simulations with varying $l_{0}$ we fix the periodicity of the lattice, $l_{0}+b$. Therefore, for large values of $l_{0}$ the base of triangles becomes small, and eventually of order $w$, strongly reducing the geometric pinning mechanism when $w \sim b$.

In the inset of Fig. 8 we show $H_{\mathrm{F}}$ versus $\theta$ for the $\phi^{4}$ wall, for different values of $l_{0}$ and constant $c=50$. The main panel of Fig. 8, shows the forward-backward asymmetry for the flat wall calculated as $H_{\mathrm{F}} / H_{\mathrm{B}}$ in comparison with the analytical prediction of Eq. (18) for a narrow domain wall $H_{\mathrm{F}} / H_{\mathrm{B}}=$ $\sin \theta$. The simulated values follow nicely the $\sin \theta$ line except for small deviations at small and large angles $\theta \sim 90^{\circ}$. These can be in part attributed to the discreteness of the lattice, which does not allow production of smooth slopes at the scale $w$ when the angle is too close to $\theta=0^{\circ}$ and $\theta=90^{\circ}$.

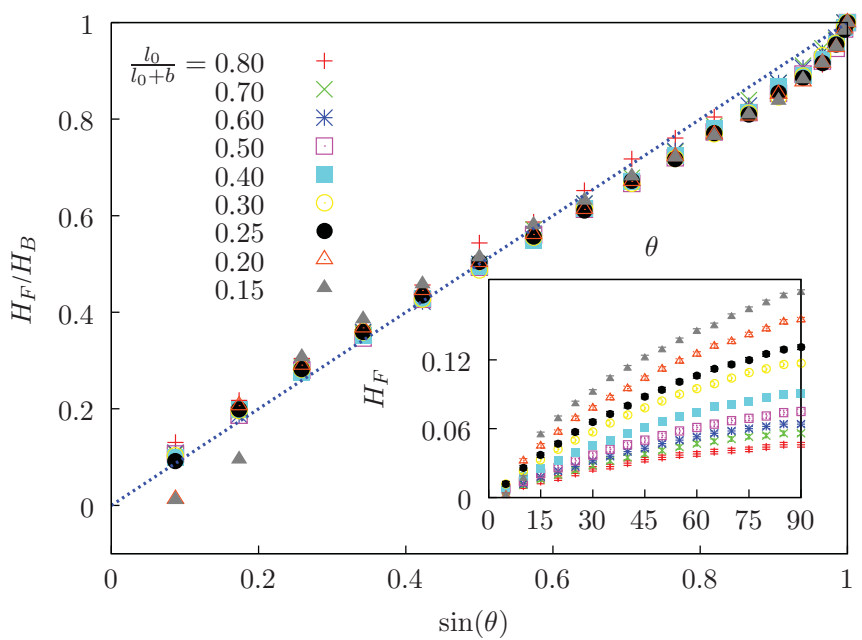

FIG. 8. (Color online) Forward-backward asymmetry for the flat $\phi^{4}$ wall with $c=50, H_{\mathrm{F}} / H_{\mathrm{B}}$ vs $\sin \theta$, calculated for different $l_{0} /\left(l_{0}+\right.$ $b$ ). The inset shows the forward depinning field, $H_{\mathrm{F}}$, for a $\phi^{4}$ domain wall, as a function of the isosceles angle, $\theta$, for different values of the vertical gap between triangles, $l_{0}$.
In short, our simulations with the $\phi^{4}$ model are consistent with the geometric estimates for a narrow wall and show how to correct the depinning fields for single arcs with a finite width, which can be relevant for experimental situations. ${ }^{24}$ The depinnings of single arcs are, on the other hand, the main building blocks for calculating all the anisotropic depinning fields and, in particular, the crossed-ratchet effect. Thus, the softening of the critical fields observed due to finite-width corrections and/or the effect of rounded tips could also be applied in a similar way to the geometric estimates of the propagation of kinked walls.

\section{B. Kinked wall: Upward and downward propagation}

\section{Upward propagation}

The depinning field for kinks can also be calculated using the basic geometry analyzed in Sec. II C. Figure 9 shows the evolution of a wall forming a kink as it is pushed upward when the field increases from $H=0$. The initial disposition of the wall is labeled as 0 . The critical field $H_{\mathrm{U}}=\sigma /\left(2 r_{\min }\right)$ for a complete depinning of the kink is given by the arc with minimal radius $r_{\min }$. For a given geometry, one has to carefully trace the trajectory of the wall, as depicted in Fig. 9, and compute the minimal radius in each step.

To step from 0 to 1 , i.e., to depin the transverse horizontal segment of the kink, is similar to the situation depicted in Fig. 4 with $\alpha=90^{\circ}-\theta$. Therefore, the corresponding critical field is $\sigma \cos \theta /(2 h)$. However, the base $b$ of the triangle can be too short for the domain wall to reach the symmetrical position described in Fig. 4. The wall is maximally tilted at an angle $\beta$ (see Fig. 5). In this situation, the corresponding angles $\theta_{1}=\theta_{2}$ in Fig. 2 are equal to $\beta$ (the angle formed with the base of the rightmost triangle in Fig. 9) and the distance between the two contact points is $l=h / \cos \beta$. The wall reaches this orientation if $H>\sigma \sin \beta / l=\sigma \sin (2 \beta) /(2 h)$. Consequently, the critical

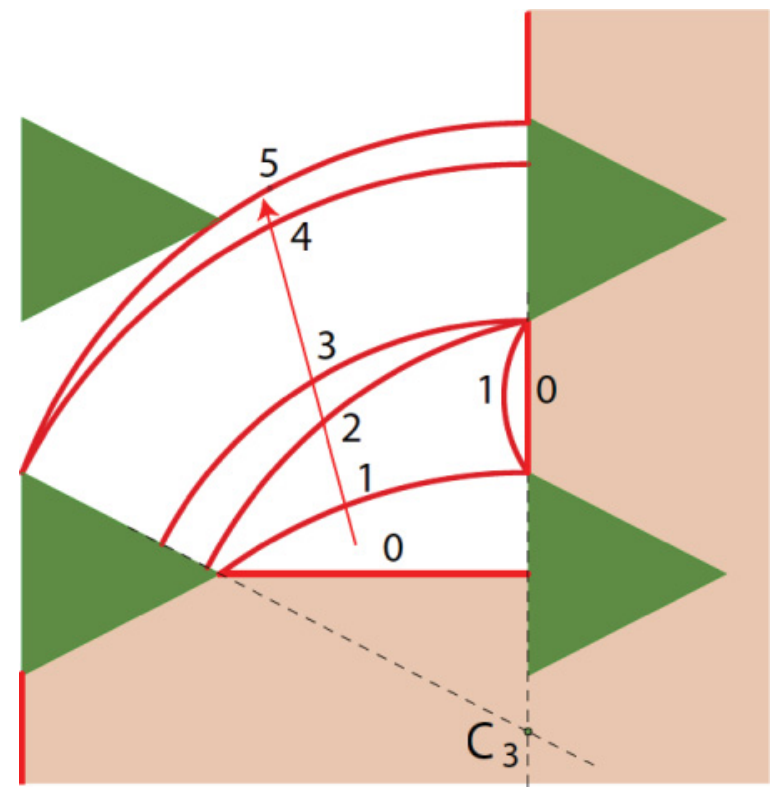

FIG. 9. (Color online) Upward motion of a kink in an elastic wall (red online). 

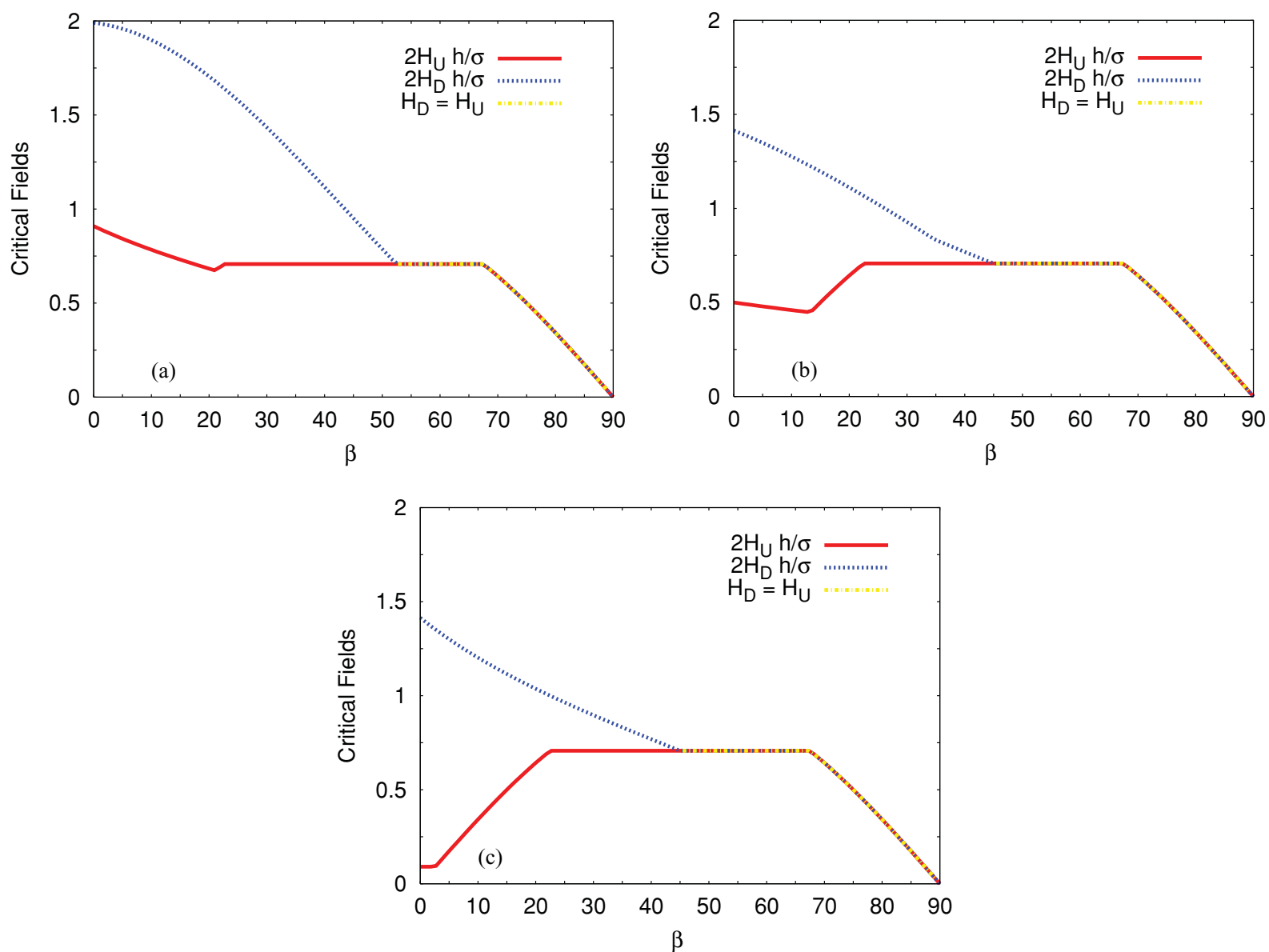

FIG. 10. (Color online) Critical fields, $H_{\mathrm{D}}$ and $H_{\mathrm{U}}$, normalized by $\sigma / 2 h$, vs $\beta$ calculated for $\theta=45^{\circ}$ for (a) $h / l_{0}=10$, (b) $h / l_{0}=1$, and (c) $h / l_{0}=0.1$. Note that for large $\beta, H_{\mathrm{D}}$ becomes equal to $H_{\mathrm{U}}$ and, therefore, a single line appears in the figures.

field to move from 0 to 1 is the minimum of these two fields, namely,

$$
\begin{aligned}
H_{1}^{\mathrm{U}} & =\frac{\sigma}{2 h} \min \{\cos (\theta), \sin (2 \beta)\} \\
& =\frac{\sigma}{2 h} \min \left\{\cos (\theta), \frac{b h}{h^{2}+(b / 2)^{2}}\right\} .
\end{aligned}
$$

The next critical arc is 3, a wall orthogonal to the contact sides of the triangles across a diagonal of the rectangular cell of triangles. The center of this arc is the point $C_{3}$, the intersection between the two prolongations of the triangles sides. The radius of arc 3 is $l_{0}+b / 2+h / \tan \theta$, and the corresponding critical field is

$$
H_{3}^{\mathrm{U}}=\frac{\sigma}{2 h}\left(\frac{1}{l_{0} / h+\tan \beta+1 / \tan \theta}\right) .
$$

Finally, one should also consider the diagonal arc 5 to complete the upward motion of the kink. However, the radius of this arc is bigger than $h_{t}+h=b /(2 \tan \theta)+h$, resulting in lower critical fields for the geometries considered in this paper. The final result for upward motion is

$$
H_{\mathrm{U}}=\max \left\{H_{1}^{\mathrm{U}}, H_{3}^{\mathrm{U}}\right\} \text {. }
$$

From the above equations, three geometrical parameters of the rectangular array of triangles are found to control the interplay between the different depinning processes of the kinked wall and, thus, the relevant critical fields: the angle $\theta$ that defines the triangle shape, the angle $\beta$ that characterizes the shape of the horizontal intertriangle region (where $\beta$ is given by $\tan \beta=b / 2 h$ ), and the ratio $h / l_{0}$ between horizontal and vertical triangle distance. This last parameter, $h / l_{0}$, is only important in the depinning of the diagonal arc 3 . Figure 10 shows the calculated $H_{\mathrm{U}}$, normalized by the scale factor $\sigma / 2 h$, as a function of $\beta$ for $\theta=45^{\circ}$ and different values of the ratio $h / l_{0}=10,1,0.1$. For large $\beta, H_{\mathrm{U}}$ is given by $H_{1}^{\mathrm{U}}$, so that it is the same in the three panels of Fig. 10. Below $\beta \simeq 22^{\circ}$, there is a crossover to $H_{\mathrm{U}}=H_{3}^{\mathrm{U}}$ indicated by the upturn in $H_{\mathrm{U}}(\beta)$ as $\beta$ decreases. It occurs at different angular positions depending on $h / l_{0}: \beta_{c}=2.6^{\circ}, 13^{\circ}$, and $21^{\circ}$ for $h / l_{0}=0.1,1$, and 10 , respectively. That is, for small $\beta$ and large $h / l_{0}$ (very anisotropic rectangular array), critical upward depinning occurs at the diagonal arc 3 in Fig. 9, whereas in the rest of the parameter space the relevant process corresponds to depinning of the transverse domain wall segment between adjacent triangles in the same row (arc 1 in Fig. 9).

\section{Downward propagation}

The first step $0 \rightarrow 1$ of the downward motion (Fig. 11) is identical to the same step in the upward motion; hence $H_{1}^{\mathrm{D}}=H_{1}^{\mathrm{U}}$. From 1 to 3 , the arc 2 has the minimal radius, which equals the distance between its center $A$ and the vertex 


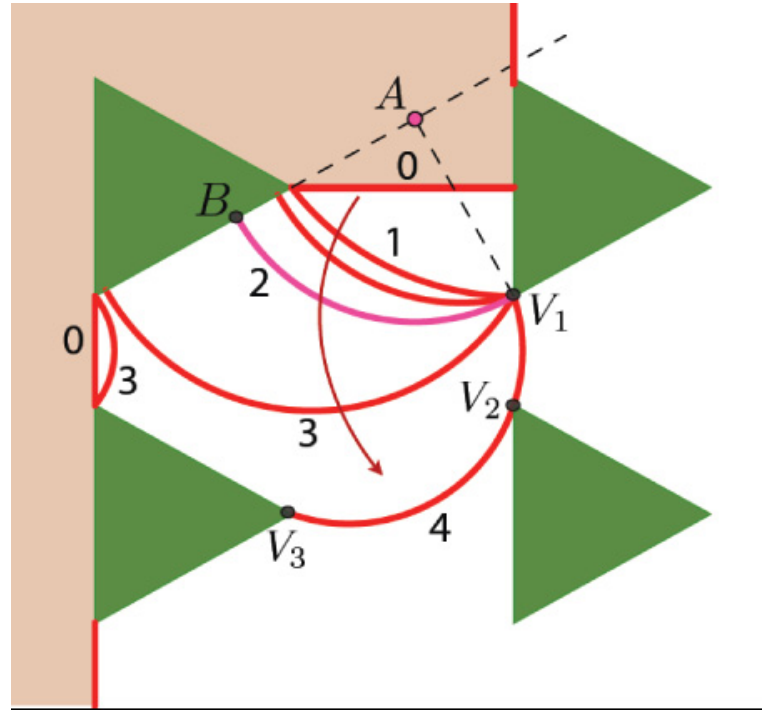

FIG. 11. (Color online) Downward motion of a kink in an elastic wall (red online).

$V_{1},\left(h+h_{t}\right) \sin \theta$. However, point $B$ can lie below or above the side of the triangle. In the first case, which occurs if $\theta>$ $45^{\circ}$, the critical field is given by the radius of arc $3,(h+$ $\left.h_{t}\right) /(2 \cos \theta)$. The second case occurs when $\theta<45^{\circ}-\beta$ and then the minimum radius, $h /[2 \cos (\beta+\theta) \cos \beta]$, occurs when point $B$ is at the vertex of the triangle. The field to reach $\operatorname{arc} 3$ can be written as

$$
H_{3}^{\mathrm{D}}=\frac{\sigma}{2 h} \begin{cases}2 \cos (\beta+\theta) \cos \beta, & \text { if } \theta<45^{\circ}-\beta, \\ \frac{1}{[\tan \beta / \tan \theta+1] \sin \theta}, & \text { if } 45^{\circ}-\beta<\theta<45^{\circ}, \\ \frac{2 \cos \theta}{\tan \beta / \tan \theta+1}, & \text { if } \theta>45^{\circ} .\end{cases}
$$

Finally, the radius of the diagonal arc 4 is

$$
r_{4}=\frac{1}{2} \sqrt{\left(h+\frac{2 l_{0} b+b^{2}}{4 h}\right)^{2}+l_{0}^{2}},
$$

yielding

$$
\begin{aligned}
H_{4}^{\mathrm{D}} & =\frac{\sigma}{2 r_{4}} \\
& =\frac{\sigma}{2 h}\left(\frac{2}{\sqrt{\left(l_{0} / h\right)^{2}+\left(1+l_{0} / h \tan \beta+\tan ^{2} \beta\right)^{2}}}\right)
\end{aligned}
$$

and

$$
H^{\mathrm{D}}=\max \left\{H_{1}^{\mathrm{U}}, H_{3}^{\mathrm{D}}, H_{4}^{\mathrm{D}}\right\} .
$$

Figure 10 shows the calculated $H_{\mathrm{D}}$, normalized by the scale factor $\sigma / 2 h$, as a function of $\beta$ for $\theta=45^{\circ}$ and different values of the ratio $h / l_{0}=10,1$, and 0.1 . For large $\beta, H_{\mathrm{D}}$ is given by $H_{1}^{\text {D }}$, i.e., depinning of the transverse horizontal wall segment, but as $\beta$ decreases $H_{3}^{\mathrm{D}}$ and $H_{4}^{\mathrm{D}}$ become more relevant. In particular, for large $h / l_{0}, H_{4}^{\mathrm{D}}$ dominates the behavior in a wide $\beta$ range. The result is that, in the low- $\beta$ range, $H_{\mathrm{D}}$ is much larger than $H_{\mathrm{U}}$, but above a certain threshold $\beta_{0}$ both fields become equal $\left(H_{\mathrm{D}}=H_{\mathrm{U}}\right)$ (for example, for $h / l_{0}=1, \beta_{0}=$ $45^{\circ}$ ). Thus, for $\beta<\beta_{0}$, upward kink propagation is easier than downward propagation so that when an alternating field of peak intensity $H$, with $H_{\mathrm{U}}<H<H_{\mathrm{D}}$, is applied to the kinked wall, it will have a net backward motion, i.e., opposite to the behavior observed in the previous section on flat wall propagations. On the other hand, for $\beta>\beta_{0}, H_{\mathrm{D}}=H_{\mathrm{U}}$, so that kink propagation is not rectified by the array of triangles.

In short, for $\theta=45^{\circ}$, as is the case in Fig. 10, whenever kinked wall propagation is asymmetric, it results in an inverted ratchet effect. This is actually the case for most of the parameter space $\left(\beta, \theta, h / l_{0}\right)$. For example, Fig. 12(a) shows the phase diagram in the $(\beta, \theta)$ plane for $h / l_{0}=1$, in which only these two regimes for domain wall propagation are found: inverted ratchet $\left(H_{\mathrm{D}}>H_{\mathrm{U}}\right)$ in the low- $\beta$ region and symmetric kink propagation in the right bottom corner of the diagram. This is a direct consequence of the maximum condition imposed in Eq. (26), as long as $H^{\mathrm{U}}=H_{1}^{\mathrm{U}}$. However, at large $h / l_{0}$, the role of arc 3 in Fig. 9 in critical upward depinning becomes more important and $H^{\mathrm{U}}$ is given by $H_{3}^{\mathrm{U}}$ in a wider $(\beta, \theta)$ region. In this case, $H_{U}$ can take any value in comparison with $H_{D}$, so that a direct ratchet effect for kinked wall motion becomes possible. An example of this situation can be seen in Fig. 12(b) for $\theta=78^{\circ}$ and $h / l_{0}=10$. Thus, the phase diagram for this very anisotropic array of triangles with $h / l_{0}=10$ [Fig. 12(c)] becomes more complex: an inverse ratchet effect $\left(H_{\mathrm{D}}>H_{\mathrm{U}}\right)$ is found in a large $(\beta, \theta)$ region in the left part of the diagram, kink motion is symmetric in the right part of the diagram $\left(H_{\mathrm{D}}=H_{\mathrm{U}}\right)$, and, finally, a direct ratchet effect (i.e., $H_{\mathrm{D}}<H_{\mathrm{U}}$ ) is found in two small regions close to the upper part of the diagram, above $\theta=65^{\circ}$. These direct ratchet regions shrink as $h / l_{0}$ decreases and disappear for $h / l_{0}<2$ due to the softening of the depinning processes of the diagonal arcs in comparison with depinning of the horizontal transverse segments.

\section{Crossed ratchets}

From the previous analysis, we have found a fundamental difference between flat and kinked wall propagation modes: $H_{\mathrm{F}} / H_{\mathrm{B}}$ is always smaller than unity, implying that flat wall propagation under an ac field will result in direct ratchet effects; in contrast, $H_{\mathrm{U}} / H_{\mathrm{D}}$ can take any value so that kinked wall motion can result either in direct or inverse rectification effects. Thus, the first condition to design an asymmetric array of defects that displays crossed-ratchet behavior is to choose a point in the phase diagram of Fig. 12 in which $H_{\mathrm{U}} / H_{\mathrm{D}}<1$. Then, in order to observe clear crossed-ratchet effects that can be useful for device applications, the interplay among the four relevant critical fields $H_{\mathrm{F}}, H_{\mathrm{B}}, H_{\mathrm{D}}$, and $H_{\mathrm{U}}$ must be taken into account.

In Fig. 13 we plot the four critical fields as a function of $h / l_{0}$ between triangles, normalized by $H_{\mathrm{B}}$ for $\beta=30^{\circ}$ and $\theta=$ $30^{\circ}$, which is similar to the geometry used in the experiments of Ref. 3, where crossed-ratchet effects were observed both experimentally and theoretically. The crossed-ratchet effect is apparent from the figure: $H_{\mathrm{F}}$ is smaller than $H_{\mathrm{B}}$, but $H_{\mathrm{U}}$ (the upward motion of the kink drives the wall backward) is larger than $H_{\mathrm{D}}$. Domain wall propagation in the array is determined by the relationships among the four critical fields: 

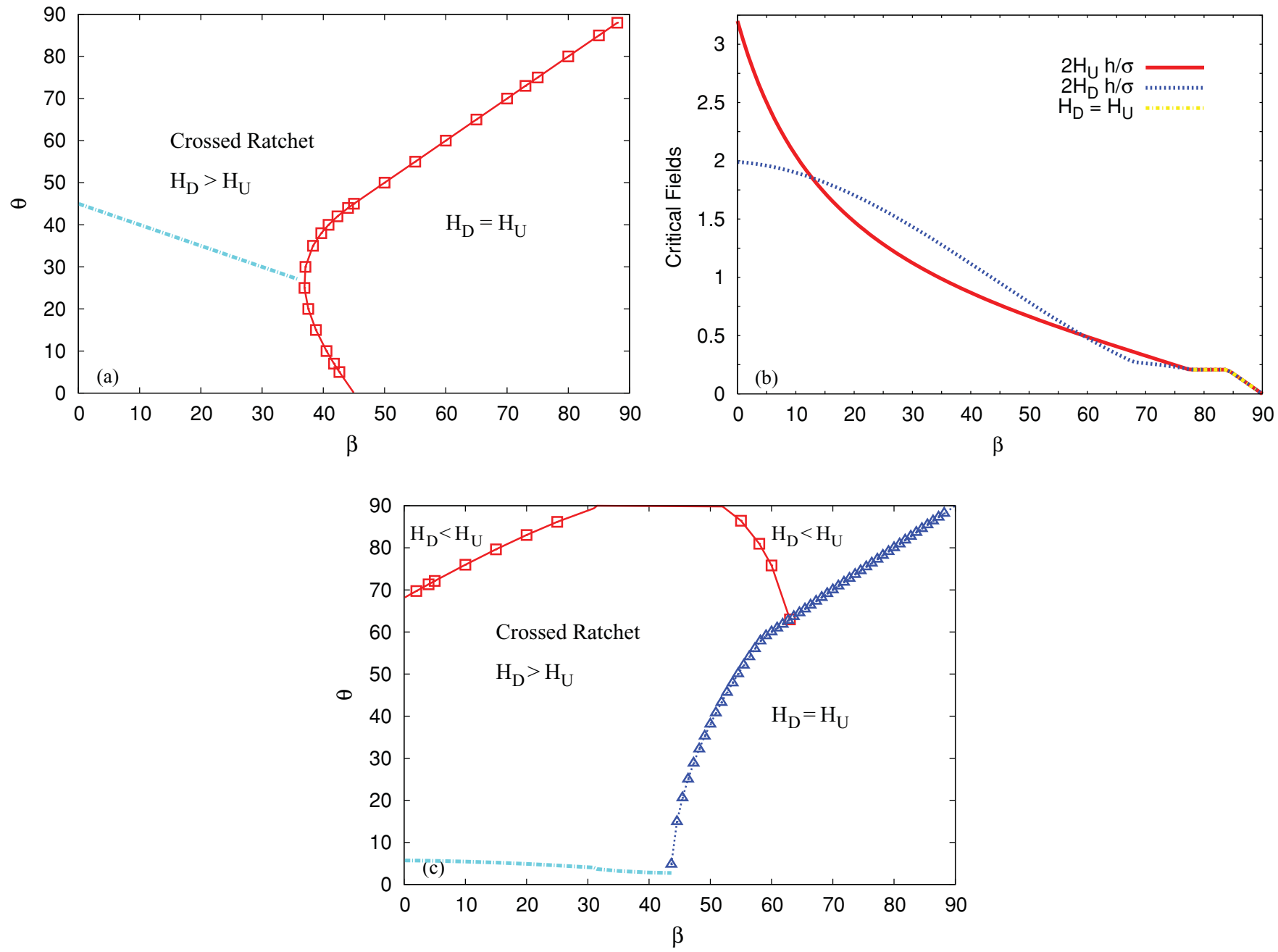

FIG. 12. (Color online) (a) Phase diagram in the $(\beta, \theta)$ plane for the different regimes for kinked wall propagation for $h / l_{0}=1$. (b) Critical fields, $H_{\mathrm{D}}$ and $H_{\mathrm{U}}$, normalized by $\sigma / 2 h$, vs $\beta$ calculated for $\theta=78^{\circ}$ for $h / l_{0}=10$. (c) Same as in (a) for $h / l_{0}=10$. Dotted lines in (a) and (c) indicate the condition $H_{\mathrm{D}}=H_{\mathrm{F}}$, below which flat wall propagation modes compete with kinked wall propagation.

for each particular array geometry (i.e., each $h / l_{0}$ value) when a domain wall is pushed in the forward direction it will depin as soon as the applied field reaches the lowest of $H_{\mathrm{F}}$ and $H_{\mathrm{D}}$; but, when a domain wall is pushed in the backward direction it will depin as soon as the applied field reaches the lowest of $H_{\mathrm{B}}$ and $H_{\mathrm{U}}$. For large $h / l_{0}$ (i.e., very rectangular array cell), both $H_{\mathrm{D}}$ and $H_{\mathrm{U}}$ take much lower values than $H_{\mathrm{B}}$ and $H_{\mathrm{F}}$, implying that two well-separated field ranges can be defined: a low field domain wall propagation dominated by kink motion (i.e., easier backward wall motion) and a high field domain wall propagation dominated by flat wall motion (i.e., easier forward wall motion). As $h / l_{0}$ is reduced below $\approx 1, H_{\mathrm{D}}$ becomes larger than $H_{\mathrm{F}}$, the interplay between flat and kinked wall propagation modes becomes more complex, and the different rectification effects cannot be clearly separated. Finally, for very close triangle lines $\left(h / l_{0}\right.$ below 0.25$), H_{\mathrm{U}}$ becomes larger than $H_{\mathrm{B}}$ and domain wall motion in the array is dominated by flat wall propagation modes.

The condition $H_{\mathrm{D}}=H_{\mathrm{F}}$ is plotted as a dotted line in Figs. 12(a) and 12(c), so that the region for well-separated kinked and flat propagation modes, i.e., clear crossed-ratchet observation, lies above this line in the $(\beta, \theta)$ plane. It can be seen that as $h / l_{0}$ increases the available parameter region for crossed ratcheting becomes wider due to the different scaling of the critical fields: $H_{\mathrm{F}}$ and $H_{\mathrm{B}}$ scale as $1 / l_{0}$, whereas $H_{\mathrm{D}}$

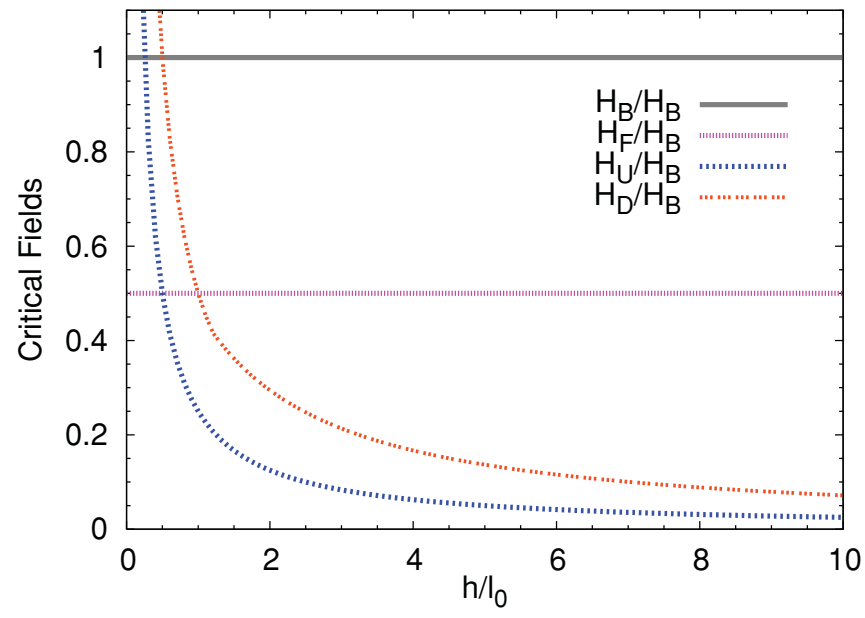

FIG. 13. (Color online) Critical fields, for $\theta=30^{\circ}$ and $\beta=30^{\circ}$, as a function of $h / l_{0}$. 
and $H_{\mathrm{U}}$ scale as $1 / h$. Thus, the design of arrays in the large- $h$ range $\left(h \gg l_{0}\right)$ appears as an important condition for a clear observation of crossed-ratchet effects that can be of use in device applications.

\section{CONCLUSIONS}

In summary, the propagation of an elastic domain wall in a two-dimensional medium has been analyzed in an arbitrary geometry defined by holes and sample boundaries. The local depinning fields for an anchored wall have been calculated as a function of boundary shape in terms of the minimal arc radius that satisfies the relevant orthogonality conditions. Then, these results have been applied to the design of 2D arrays of asymmetric holes with broken $Y$-axis reflection symmetry that can display crossed-ratchet effects (i.e., direct ratchet for forward and backward flat wall propagation and inverted ratchet for upward and downward kink propagation).

For a rectangular array of triangles, flat wall propagation is found to be always asymmetric, resulting in a direct ratchet effect controlled by triangle shape (angle $\theta$ ) and intertriangle vertical distance $\left(l_{0}\right)$. Corrections due to finite domain wall width and/or rounded triangle tips, which could be relevant in actual patterned arrays of holes, produce a global softening of the critical fields but do not alter significantly forward-backward asymmetry. On the other hand, upward and downward kink propagation can display any asymmetry and depends not only on triangle shape but also on the shape of the horizonal intertriangle region (angle $\beta$ ) and on the array vertical-horizontal anisotropy $\left(h / l_{0}\right)$. The array geometry needed for the observation of crossed-ratchet effects has been determined by considering the different wall propagation modes relevant in the different points of the $(\beta, \theta)$ plane. Anisotropic arrays with large $h / l_{0}$ are found to be optimum for the observation of clear crossed-ratchet effects.

\section{ACKNOWLEDGMENTS}

This work is supported by SeCyT-UNCórdoba and CONICET, Argentina, Spanish MICINN (FIS2008-06249 and HP2008-0032) and Asturias FICYT (IB08-106), and Grants MOSAICO (Spain) and MODELICO-CM (Comunidad de Madrid, Spain). J. A. Capitán acknowledges funding by a contract from Comunidad de Madrid and Fondo Social Europeo. A.B. Kolton acknowledges ANPCYT (Grant No. PICT2007886, Argentina) and Universidad de Barcelona, Ministerio de Ciencia e Innovación (Spain), and Generalitat de Catalunya for partial support through the I3 program.

\section{APPENDIX A: FROM FIELD EQUATIONS TO ELASTIC INTERFACES}

In this Appendix we construct an approximate stationary solution of Eq. (1) around a given curve in the plane, defined as $[x(s), y(s)]$, with $s$ a real number taking values in some interval.

The interface of the desired solution is centered around the line $[x(s), y(s)]$, i.e., $\phi(x(s), y(s))=0$, and the field approaches the stable values \pm 1 as we move away from the line. We then construct the solution using the signed distance function, $g(x, y)$, whose absolute value is the distance of a point $(x, y)$ to the line $[x(s), y(s)]$. Obviously, $g(x(s), y(s))=0$ for all $s$. One less obvious property is that the gradient of the distance function is unitary all over the plane. In other words, the distance function obeys the eikonal equation

$$
\left[\partial_{x} g(x, y)\right]^{2}+\left[\partial_{y} g(x, y)\right]^{2}=1 .
$$

Now we choose the following form for the field: $\phi(x, y)=$ $f(g(x, y))$. Introducing this ansatz in the stationary $\phi^{4}$ equation and making use of Eq. (A1), we get

$$
c f^{\prime \prime}(g)+c f^{\prime}(g) \nabla^{2} g+\epsilon_{0}\left[f(g)-f(g)^{3}\right]=0 .
$$

Our first approximation consists of neglecting $f^{\prime}(g(x, y)) \nabla^{2} g(x, y)$ in the above equation. The Laplacian of the distance function is inversely proportional to the curvature radius of the line $[x(s), y(s)]$. Therefore, our approximation is valid for interfaces with a curvature radius much larger than its width. Then, Eq. (A2) reduces to

$$
c f^{\prime \prime}(g(x, y))+\epsilon_{0}\left[f(g(x, y))-f(g(x, y))^{3}\right]=0
$$

and the general solution reads $f(z)=\tanh \left[\left(z-z_{0}\right) / w\right]$ with

$$
w=\sqrt{\frac{2 c}{\epsilon_{0}}} .
$$

The field $\phi$ is then given by

$$
\phi(x, y)=\tanh \left[\frac{g(x, y)}{w}\right],
$$

where we have absorbed the constant $z_{0}$ in the function $g$ to center the wall along the line $[x(s), y(s)]$ where $g$ vanishes. Equation (A5) is well known as the field corresponding to a single wall in the one-dimensional $\phi^{4}$ model.

To calculate the energy of the solution given by Eq. (A5), it is convenient to use as coordinates the distance $z$ to the center of the interface and $s$, the parameter defining this center. These new coordinates $(s, z)$ are related with the Cartesian coordinates $(x, y)$ as $[x(s, z), y(s, z)]$, obeying

$$
g(x(s, z), y(s, z))=z \quad \forall s, z .
$$

The $z=0$ contour line is our initial curve $[x(s), y(s)]$. The Jacobian of this change of coordinates can be calculated by differentiating Eq. (A6) with respect to $s$ and $z$, respectively, yielding

$$
d x d y=\sqrt{\dot{x}^{2}+\dot{y}^{2}} d s d z,
$$

where the dot denotes differentiation with respect to $s$. This is in fact the product of the elementary length of the contour line $\sqrt{\dot{x}^{2}+\dot{y}^{2}} d s$ times $d z$.

We can now calculate the energy of the wall by inserting the solution (A5) in Eq. (2). With the change of variables $(x, y) \rightarrow(s, z)$, the energy reduces to

$$
E=\int d z L(z)\left[U(f(z))+H f(z)+\frac{c}{2} f^{\prime}(z)^{2}\right],
$$

where $f(z)=\tanh (z / w)$ and

$$
L(z)=\int d s \sqrt{\dot{x}(s, z)^{2}+\dot{y}(s, z)^{2}}
$$


is the total length of the contour line $g(x, y)=z$ in the restricted geometry of the problem. In particular, $L(0)$ is the length of our starting curve $[x(s), y(s)]$ defining the center of the interface. If the interface is narrow, we can approximate $L(z) \simeq L(0)$ for those $z$ where $U(f(z))$ is significantly different from zero, i.e., around the center of the interface. Finally, the energy due to the external field $H$ can be estimated by replacing $f(z)$ by a step function $2 \theta(z)-1$ in the term $H f(z)$. With these assumptions, the energy becomes

$$
E=\sigma L(0)-2 H A
$$

where $A$ is the area at one side of the center of the interface $[x(s), y(s)]$, and

$$
\sigma=\int_{-\infty}^{\infty}\left[U(f(z))+\frac{c}{2} f^{\prime}(z)^{2}\right]=\frac{\sqrt{8 \epsilon_{0} c}}{3}
$$

is the energy of the interface per unit of length. These are the expressions yielding (5) in the main text.

\section{APPENDIX B: EQUILIBRIUM SHAPE}

Our next step is to calculate the equilibrium profile of an interface segment. The energy of an interface segment is a function of both its shape and the location of its ends or contact points. In order to find the possible metastable states of the segment we need to minimize this energy with the constraint (8).

We describe the wall as a line given by $y(x)$, anchored to the border at points $\left(x_{1}, y_{1}\right)$ on the left and $\left(x_{2}, y_{2}\right)$ on the right (see Fig. 14). The energy of the interface is given by

$$
E=\int_{x_{1}}^{x_{2}}\left[\sigma \sqrt{1+y^{\prime}(x)^{2}}-2 H y(x)\right] d x,
$$

and hence the Euler-Lagrange equation is

$$
\frac{d}{d x} \frac{y^{\prime}}{\sqrt{1+\left(y^{\prime}\right)^{2}}}+\frac{2 H}{\sigma}=0 .
$$

We have to solve this equation by imposing the orthogonality condition at the contact points (which are otherwise free).

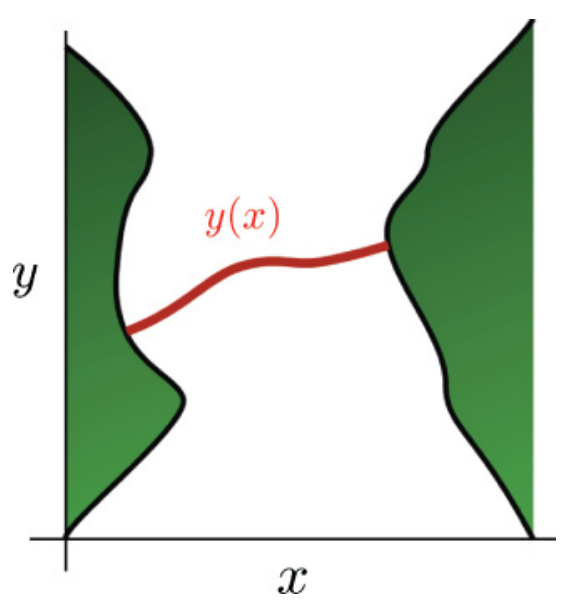

FIG. 14. (Color online) The elastic wall (red online) between two boundaries (vertical black curves) is parametrized as $y(x)$ to solve the variational problem.

One integration of (B2) gives

$$
\frac{y^{\prime}(x)}{\sqrt{1+y^{\prime}(x)^{2}}}=-\frac{x-x_{0}}{r},
$$

where $x_{0}$ is a constant and $r=\sigma /(2 H)$. From (B3) we get

$$
y^{\prime}(x)= \pm \frac{x-x_{0}}{\sqrt{r^{2}-\left(x-x_{0}\right)^{2}}},
$$

and a second integration yields

$$
y(x) \pm \sqrt{r^{2}-\left(x-x_{0}\right)^{2}}=y_{0},
$$

which, written as $\left(x-x_{0}\right)^{2}+\left(y-y_{0}\right)^{2}=r^{2}$, reveals itself as the arc of a circumference of radius $r$ and center $\left(x_{0}, y_{0}\right)$. This is in fact the Laplace law in two dimensions, relating the pressure difference to the local curvature of an elastic interface at equilibrium. It is however important to notice that this equilibrium shape is independent of the boundary conditions. We can therefore impose these conditions by looking for an arc of radius $r$ which intersects orthogonally with the two boundaries, as we do in Sec. II C using basic geometric arguments and in Sec. II D in an analytical manner.
${ }^{1}$ A.-L. Barabasi and H. E. Stanley, in Fractal Concepts in Surface Growth (Cambridge University Press, Cambridge, 1995).

${ }^{2}$ G. Mahmud, C. J. Campbell, K. J. M. Bishop, Y. A. Komarova, O. Chaga, S. Soh, S. Huda, K. Kandere-Grzybowska, and B. A. Grzybowski, Nature Phys. 5, 606 (2009).

${ }^{3}$ A. Pérez-Junquera, V. I. Marconi, A. B. Kolton, L. M. AlvarezPrado, Y. Souche, A. Alija, M. Vélez, J. V. Anguita, J. M. Alameda, J. I. Martín, and J. M. R. Parrondo, Phys. Rev. Lett. 100, 037203 (2008).

${ }^{4}$ S. Lemerle, J. Ferré, C. Chappert, V. Mathet, T. Giamarchi, and P. Le Doussal, Phys. Rev. Lett. 80, 849 (1998).

${ }^{5}$ L. Krusin-Elbaum, T. Shibauchi, B. Argyle, L. Gignac, and D. Weller, Nature (London) 410, 444 (2001)

${ }^{6}$ V. Repain, M. Bauer, J.-P. Jamet, J. Ferré, A. Mougin, C. Chappert, and H. Bernas, Europhys. Lett. 68, 460 (2004).
${ }^{7}$ F. Cayssol, D. Ravelosona, C. Chappert, J. Ferré, and J. P. Jamet, Phys. Rev. Lett. 92, 107202 (2004).

${ }^{8}$ P. J. Metaxas, J. P. Jamet, A. Mougin, M. Cormier, J. Ferré, V. Baltz, B. Rodmacq, B. Dieny, and R. L. Stamps, Phys. Rev. Lett. 99, 217208 (2007).

${ }^{9}$ T. Tybell, P. Paruch, T. Giamarchi, and J. M. Triscone, Phys. Rev. Lett. 89, 097601 (2002).

${ }^{10}$ P. Paruch, T. Giamarchi, and J. M. Triscone, Phys. Rev. Lett. 94, 197601 (2005).

${ }^{11}$ W. Kleemann, Annu. Rev. Mater. Res. 37, 415 (2007).

${ }^{12}$ D. Wilkinson and J. F. Willemsen, J. Phys. A 16, 3365 (1983).

${ }^{13}$ S. Moulinet, C. Guthmann, and E. Rolley, Eur. Phys. J. E 8, 437 (2002).

${ }^{14}$ E. Bouchaud, J. P. Bouchaud, D. S. Fisher, S. Ramanathan, and J. R. Rice, J. Mech. Phys. Solids 50, 1703 (2002). 
${ }^{15}$ M. Alava, P. K. V. V. Nukalaz, and S. Zapperi, Adv. Phys. 55, 349 (2006).

${ }^{16}$ R. P. Cowburn, A. O. Adeyeye, and J. A. C. Bland, Appl. Phys. Lett. 70, 2309 (1997).

${ }^{17}$ A. Pérez-Junquera, G. Rodríguez-Rodríguez, M. Vélez, J. I. Martín, H. Rubio, and J. M. Alameda, J. Appl. Phys. 99, 033902 (2006).

${ }^{18}$ G. Rodríguez-Rodríguez, J. L. Menéndez, A. Hierro-Rodríguez, A. Pérez-Junquera, N. Montenegro, D. Ravelosona, J. M. Alameda, and M. Vélez, J. Phys. D 43, 305002 (2010).

${ }^{19}$ T. Ono, H. Miyajima, K. Shigeto, and T. Shinjo, Appl. Phys. Lett. 72, 1116 (1998)

${ }^{20}$ S. Savelév, A. L. Rakhmanov, and F. Nori, Phys. Rev. B 74, 024404 (2006).

${ }^{21}$ N. Hayashi et al., IEEE Trans. Magn. 8, 370 (1972).

${ }^{22}$ D. A. Allwood, G. Xiong, and R. P. Cowburn, Appl. Phys. Lett. 85, 2849 (2004).

${ }^{23}$ L. K. Bogart, D. Atkinson, K. O'Shea, D. McGrouther, and S. McVitie, Phys. Rev. B 79, 054414 (2009).
${ }^{24}$ A. Alija, A. Pérez-Junquera, G. Rodríguez-Rodríguez, M. Vélez, V. I. Marconi, A. B. Kolton, J. V. Anguita, J. M. Alameda, J. M. R. Parrondo, and J. I. Martin, J. Phys. D 42, 045001 (2009).

${ }^{25}$ See, for instance, P. M. Chaikin and T. C. Lubensky, Principles of Condensed Matter Physics (Cambridge University Press, Cambridge, 1995).

${ }^{26}$ N. R. Quintero, A. Sánchez, and F. G. Mertens, Phys. Rev. E 62, 5695 (2000).

${ }^{27}$ The possibility that a moving solution at field $H$ could "get around" a static solution in front of it with local depinning field $H_{c}>H$ is ruled out for an elastic interface with overdamped dynamics by virtue of the "noncrossing rule" [see A. A. Middleton, Phys. Rev. Lett. 68, 670 (1992); A. Rosso and W. Krauth, Phys. Rev. E 65, 025101(R) (2002)].

${ }^{28}$ W. H. Press, S. A. Teukolsky, W. T. Vetterling, and B. P. Flannery, Numerical Recipes: The Art of Scientific Computing, 3rd ed. (Cambridge University Press, Cambridge, 2007). 